\title{
In vitro cytotoxicity of two novel oral formulations of Amphotericin B (iCo-009 and iCo- 010) against Candida albicans, human monocytic and kidney cell lines
}

Carlos G Leon ${ }^{1 \dagger}$, Jinkyung Lee ${ }^{1 \dagger}$, Karen Bartlett ${ }^{2}$, Pavel Gershkovich', Ellen K Wasan ${ }^{3}$, Jinying Zhao ${ }^{1}$, John G Clement ${ }^{4}$ and Kishor M Wasan ${ }^{1 *}$

\begin{abstract}
Background: Invasive fungal infections such as candidiasis constitute an increasingly important medical problem. Drugs currently used for the treatment of candidiasis include polyenes (such as Amphotericin B) and azoles. Amphotericin B (AmpB) presents several limitations such as its nephrotoxicity and limited solubility. We have developed two novel lipid-based AmpB formulations which in vivo show less nephrotoxicity and enhanced solubility compared to Fungizone ${ }^{\mathrm{TM}}$ a commercial AmpB formulation.

The purpose of this study was to determine the cytotoxicity of Fungizone ${ }^{\mathrm{TM}}$, Ambisome $^{\mathrm{TM}}$ and two novel AmpB formulations (iCo-009 and iCo-010) against Candida albicans, human kidney (293T) cells and monocytic (THP1) cells.

Methods: Cell cytotoxicity to the AmpB formulations was evaluated by MTS and LDH assays. In vitro anti-Candida albicans activity was assessed after a $48 \mathrm{~h}$ drug incubation.

Results: None of the AmpB formulations tested showed cytotoxicity against 293T cells. In the case of THP1 cells only Fungizone $\mathrm{TM}^{\mathrm{TM}}$ and Ambisome ${ }^{\mathrm{TM}}$ showed cytotoxicity at $500 \mathrm{\mu g} / \mathrm{L}(\mathrm{n}=4-10, \mathrm{p}<0.05)$.

The calculated EC50 to Candida albicans for the different formulations was as follows: $26.8 \pm 2.9$ for iCo-010, $74.6 \pm$ 8.9 for iCo-009, $109 \pm 31$ for Ambisome ${ }^{\mathrm{TM}}$ and $87.1 \pm 22$ for Fungizone ${ }^{\mathrm{TM}}$ ( $\mu \mathrm{g}$ of AmpB/L, $\mathrm{n}=6-12, \mathrm{p}<0.05$ ).

Conclusions: The AmpB formulations analyzed were not cytotoxic to 293T cells. Cytotoxicity in THP1 cells was observed for Fungizone ${ }^{\mathrm{TM}}$ and $\mathrm{Ambisome} \mathrm{TM}^{\mathrm{TM}}$, but not with the novel AmpB formulations. iCo-010 had higher efficacy compared to other three AmpB formulations in the Candida albicans model.

The absence of cytotoxicity as well as its higher efficacy for the Candida model compared to Fungizone ${ }^{\mathrm{TM}}$ and Ambisome ${ }^{\mathrm{TM}}$ suggest that $\mathrm{iCo}-010$ has potential in treating candidiasis.
\end{abstract}

Keywords: Amphotericin B, Candida, cytotoxicity, monocytes.

\section{Background}

Invasive fungal infections constitute an increasingly important medical problem due to the growth of immunodeficient populations, the development of antifungal resistance and limitations in the efficacy and toxicity of

\footnotetext{
* Correspondence: kwasan@mail.ubc.ca

† Contributed equally

'Division of Pharmaceutics and Biopharmaceutics, Faculty of Pharmaceutical Sciences, The University of British Columbia, Vancouver British Columbia, V6T 1Z3, Canada

Full list of author information is available at the end of the article
}

current antifungals [1]. Candida species are the most common cause of nosocomial invasive mycosis and are the leading cause of related mortality [2]. An increasing rate of candidaemia [3] as well as the emergence of drug resistant strains [4] support the efforts in discovering novel therapeutic approaches [1].

Drugs currently used for the treatment of candidiasis include polyenes (such as Amphotericin B AmpB), azoles (fluconazole), echinocandins and flucytosine. Limited therapeutic efficiency and drug resistance have led

\section{Biomed Central}

(c) 2011 Leon et al; licensee BioMed Central Ltd. This is an Open Access article distributed under the terms of the Creative Commons Attribution License (http://creativecommons.org/licenses/by/2.0), which permits unrestricted use, distribution, and reproduction in any medium, provided the original work is properly cited. 
to consider new therapeutic approaches, in particular the use of new analogs of existing drugs [1].

Amphotericin B (AmpB) is a treatment of choice for systemic fungal infections [5]. The mechanism of action of AmpB involves its binding to ergosterol in the fungal cell membrane, producing pores in the membrane which leads to ion loss and cell death [6]. Recently it has been described that AmpB can also elicit cell death through the induction of a strong oxidative burst [7]. Acquired resistance to Amphotericin B in Candida species, though rare, has been reported previously $[8,9]$. This AmpB resistance has been correlated with decreased levels of ergosterol in the plasma membrane and has been accompanied with azole resistance $[10,11]$.

One of the major limitations associated with $A m p B$ is its nephrotoxicity [12]. Another issue related to AmpB is its poor solubility that limits its route of administration [13]. The development of an effective, safe, and inexpensive oral formulation of amphotericin B would have many applications for the treatment of fungal diseases. The new oral amphotericin B formulations developed by our group, iCo-009 and iCo-010, show efficacy in vivo against leishmaniasis $[14,15]$, aspergillosis and candidiasis [16].

The purpose of the present study was to determine if four different formulations of AmpB (iCo-009, iCo-010, Fungizone $\mathrm{e}^{\mathrm{TM}}$ and Ambisome ${ }^{\mathrm{TM}}$ ) were cytotoxic in human monocytic, human kidney cells and Candida albicans.

\section{Results and Discussion}

The first objective of our study was to determine the AmpB-induced cytotoxicity in two human cell lines, 293T kidney and THP1 monocytic cells. No evidence of cytotoxicity was found in any of the three AmpB formulations used (Fungizone ${ }^{\mathrm{TM}}$, iCo-009 and $\mathrm{iCo}-010$ ) at the doses tested (Figure 1, n $=4$ ) using a respiration assay (MTS) in the kidney 293T cells. The vehicle controls for both iCo-009 and iCo-010 did not show a toxic effect (data not shown). At the highest AmpB dose analyzed $(10,000 \mu \mathrm{g} / \mathrm{L})$, there was a reduction of $20 \%$ in the respiration rate of the cells treated with Fungizone ${ }^{\mathrm{TM}}$, however, it didn't reach statistical significance. In terms of cytotoxicity in THP1 monocytic cells of the four AmpB formulations at the doses analyzed (0 to $500 \mu \mathrm{g} /$ L), differences were shown between the MTS (Figure 2a, $\mathrm{n}=4-10$ ) and LDH (Figure $2 \mathrm{~b}, \mathrm{n}=6$ ) results. No changes in viability were found with the LDH assay at any of the AmpB concentrations analyzed. The LDH results were corrected by the amount of protein in each treatment group. There was also no difference in protein content in the different treatment groups. However, the Normalized MTS results exhibited a reduced respiration rate at $500 \mu \mathrm{g} / \mathrm{L}$ for both Fungizone $\mathrm{TM}^{\mathrm{TM}}(0.71 \pm 0.04)$ and Ambisome $^{\mathrm{TM}}(0.72 \pm 0.17)$ compared to controls (1.00).

Previously, others [17] have described a difference in the degree of cellular activation by AmpB in the 293 and THP1 cell lines. This disparity was accounted by the differential expression of the TLR2, highly expressed in THP1 cells and expressed at very low levels in 293 cells. The new oral formulations, iCo-009 and iCo-010, did not show any evidence of toxicity at the highest concentration analyzed in $293 \mathrm{~T}$ cells $(10,000 \mu \mathrm{g} / \mathrm{L}$, Figure 1). The limited TLR2 expression in 293T cells may explain this lack of cytotoxicity that was observed even at large doses of AmpB compared with a cytotoxic effect of AmpB in THP1 cells at a lower concentration (500 $\mu \mathrm{g} / \mathrm{L})$.

The difference in cytotoxicity found in THP1 cells between the LDH and MTS assays could be accounted by the fact that the MTS assay relies on the accessibility of the substrate to the respiratory machinery of the cell (i.e. expression and activity of transporters). In the case of LDH there is no active transport factor(s) as it is an index of the cell breakdown and the release of LDH enzyme to the medium. The drug concentration that elicits toxicity in the THP1 monocytes with Fungizone ${ }^{\mathrm{TM}}$ and Ambisome ${ }^{\mathrm{TM}}$ can be compared with the serum drug levels obtained in patients. After a standard dose of AmpB $(0.3 \mathrm{mg} / \mathrm{kg}$ on days 1 and 2 followed by increasing doses to $0.5 \mathrm{mg} / \mathrm{kg}$ ) for patient therapy, the expected serum AmpB levels can reach $200 \mu \mathrm{g} / \mathrm{L}$ [18]. Few studies have assessed the AmpB-induced cytotoxicity in monocytes and macrophages. Sesana et al., [19] examined the in vitro activity of $\mathrm{AmpB}$ cochleates against Leishmania chagasi and the cytotoxic effect on mouse peritoneal macrophages using AmpB deoxycholate (Fungizone $^{\mathrm{TM}}$ ) as a control. They found that the AmpB deoxycholate (Fungizone ${ }^{\mathrm{TM}}$ ) was cytotoxic to mouse peritoneal macrophages at a concentration of $1250 \mu \mathrm{g} / \mathrm{L}$, while the AmpB cochleates were not toxic at that AmpB concentration.

The second objective of our study was to determine the efficacy of the novel AmpB formulations in an in vitro Candida albicans model. Unfortunately, frequently there is limited correlation between in vitro susceptibility determination and patient response [20]. However, use of RPMI medium supplemented with dextrose showed improvement in assessing Candida albicans antibiotic susceptibility [21]. Thus this methodology was used in the present study. The results (Figure 3 and Table 1) showed that iCo-010 had a lower EC50 than Fungizone ${ }^{\mathrm{TM}}$, Ambisome ${ }^{\mathrm{TM}}$ and $\mathrm{iCo}-009$ for this ATCC Candida albicans reference strain $(\mathrm{n}=6-12$ experiments). The EC50 calculated for AmpB (as Fungizone $^{\mathrm{TM}}$ ) for the Candida albicans reference strain 18804 


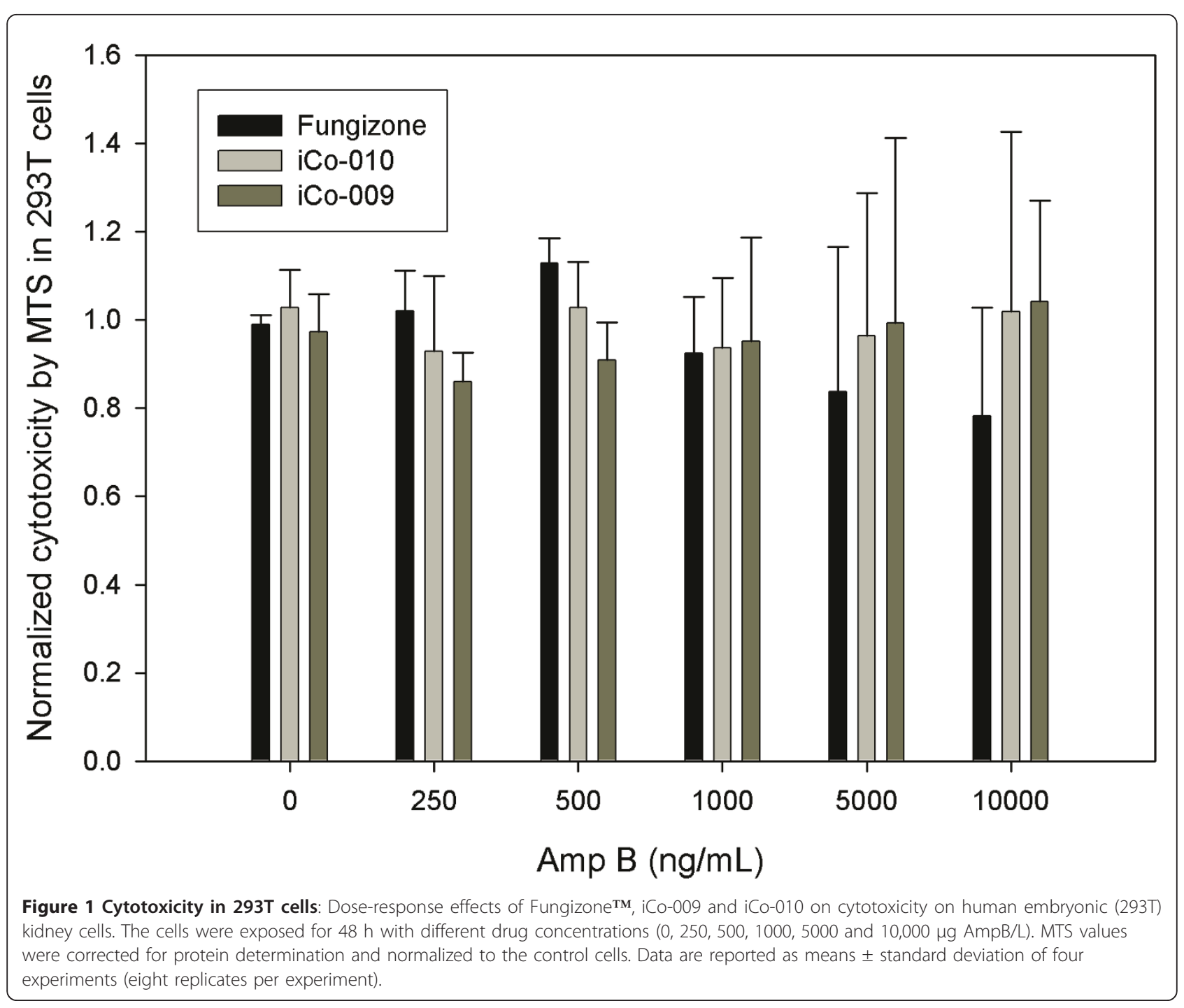

that was assessed using this system is similar to that previously obtained for this strain in an Etest [22].

iCo-009 was previously compared to Abelcet ${ }^{\circledR}$ in an in vivo study in Candida albicans-infected male rats [16]. In that report, $\mathrm{iCo}-009$ exhibited significant reduction in viable colony forming units of Candida from kidney homogenates compared to Abelcet ${ }^{\circledR}$.

\section{Conclusions}

We have tested the cytotoxicity and efficacy of four AmpB formulations in two human cell lines and a Candida albicans model, respectively. Fungizone ${ }^{\mathrm{TM}}$ and Ambisome ${ }^{\mathrm{TM}}$, but not iCo-009 and iCo-010, show cytotoxicity in human monocytes at a concentration of 500 $\mu \mathrm{g} / \mathrm{L}$. iCo-010 shows a higher efficacy compared to iCo009, Ambisome ${ }^{\mathrm{TM}}$ and Fungizone ${ }^{\mathrm{TM}}$ in the Candida albicans model. The lack of toxicity and efficacy of iCo010, a novel oral AmpB formulation, warrant future in vivo Candida albicans studies to determine its therapeutic index.

\section{Materials and methods}

\subsection{Novel lipid oral Amphotericin B formulations}

The description of the composition and development of the two novel lipid oral Amphotericin B formulations can be found elsewhere iCo-009 [16] and iCo-010 [15]. AmpB concentration was analyzed by HPLC as previously described [16]. For the cytotoxicity and efficacy studies we used as negative controls medium alone and medium containg the vehicle controls for both iCo-009 and iCo-010. No cytotoxicity was found when any of these controls were used (data not shown).

\subsection{Cytotoxicity assay on $293 \mathrm{~T}$ cells}

Human embryonic kidney cells (293T ATCC CRL 11268) were kindly provided by Dr JS Hill (St Paul's 

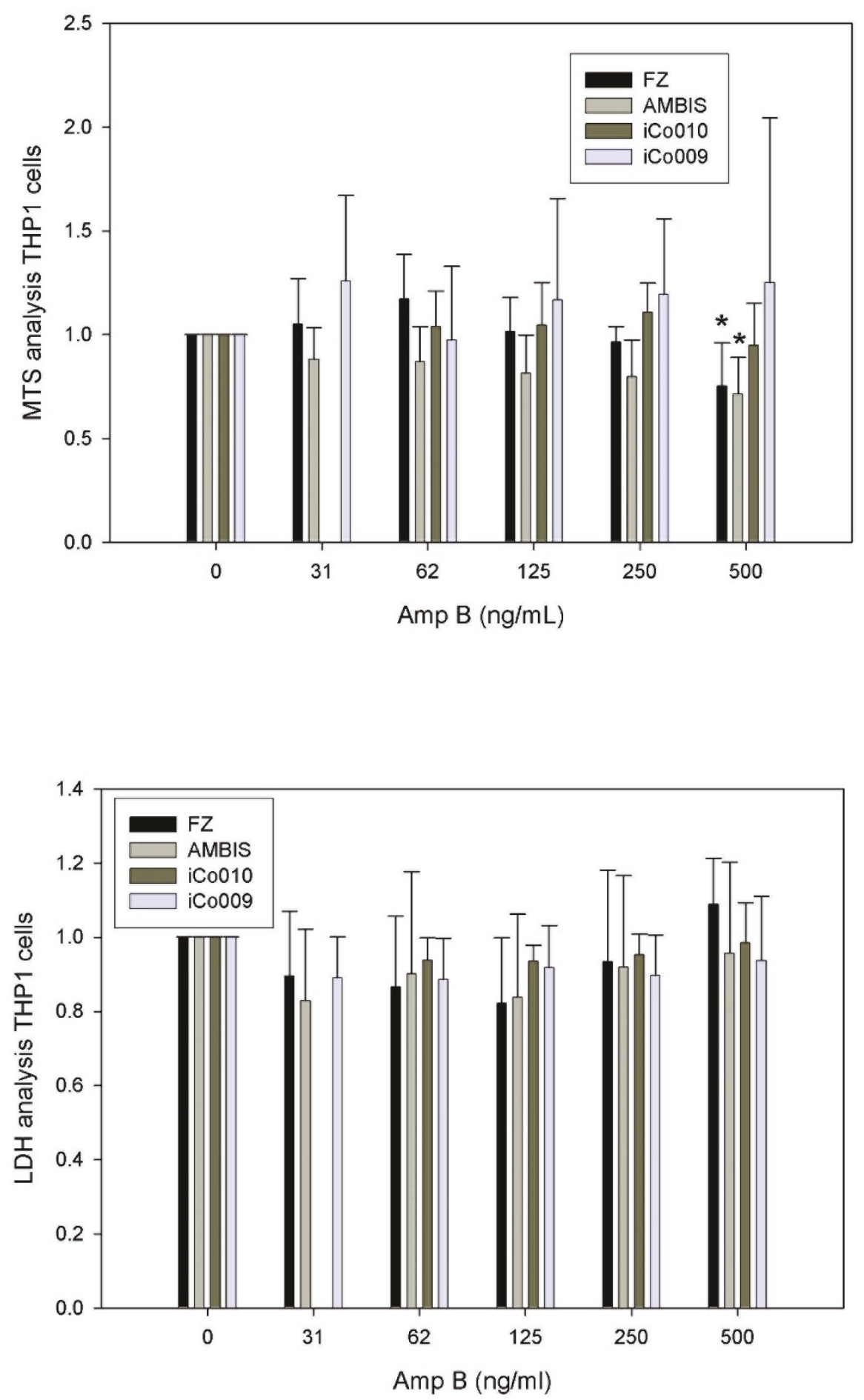

Figure 2 Dose-response effects of Fungizone ${ }^{\mathrm{TM}}$, Ambisome ${ }^{\mathrm{TM}}$, iCo-009 and iCo-010 on cytotoxicity in human monocytic (THP1) cells as assessed using an MTS assay (Figure 2a). The cells were exposed for $48 \mathrm{~h}$ with different drug concentrations $(31.25,62.5,125,250$ and $500 \mu \mathrm{g} \mathrm{AmpB/L)}$. The cells were washed and incubated with the MTS reagent for $90 \mathrm{~min}$. Data are reported as means \pm standard deviation of four to ten experiments, ${ }^{*} p<0.05$, eight replicates per experiment). Dose-response effects of Fungizone ${ }^{\mathrm{TM}}$, Ambisome ${ }^{\mathrm{TM}}$, iCo-009 and iCo-010 on cytotoxicity in human monocytic (THP1) cells as assessed using an LDH assay (Figure $\mathbf{2 b}$ ). The cells were exposed for $48 \mathrm{~h}$ with different drug concentrations $(31.25,62.5,125,250$ and $500 \mu \mathrm{g} \mathrm{AmpB/L)}$. An aliquot of the medium was incubated with the LDH reagent. Data are reported as means \pm standard deviation of six experiments (four replicates per experiment). 


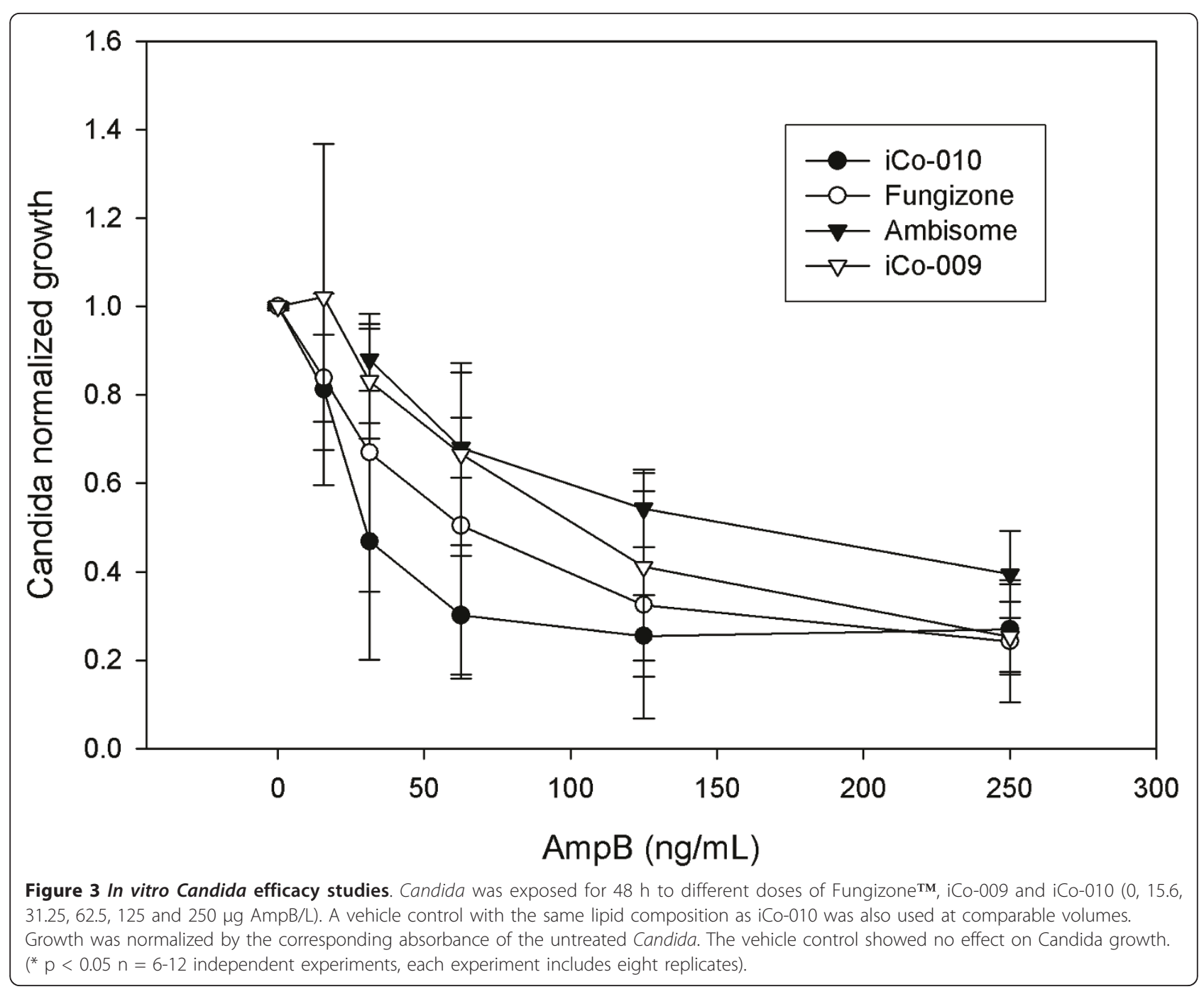

Hospital, UBC, Vancouver, BC). Cells were grown in complete DMEM without phenol red at $37^{\circ} \mathrm{C}\left(5 \% \mathrm{CO}_{2}\right)$ in T75 flasks. At near confluency, the cells were trypsinized and transferred to either additional flasks or to 96-well plates (previously coated with Poly L-lysine and seeded at a density of 10,000 cells/well). Twenty four hours post seeding, the adherent cells were washed and incubated with different concentrations of AmpB formulations (250 to $10,000 \mu \mathrm{g} / \mathrm{L}$ of $\mathrm{AmpB}$ ) for $48 \mathrm{~h}$. Percent

Table 1 EC50 for AmpB formulations (mean \pm SD, expressed in $\mu \mathrm{g} / \mathrm{L}$ of $\mathrm{AmpB}, \mathrm{n}=6-12, * \mathrm{p}<0.05$ ) tested in the Candida model

\begin{tabular}{cc}
\hline & Mean \pm SD \\
\hline Fungizone $^{\text {TM }}$ & $87.1 \pm 22$ \\
\hline Ambisome $^{\text {TM }}$ & $109 \pm 31$ \\
\hline iCo-009 & $74.6 \pm 8.9$ \\
\hline iCo-010 & $26.8 \pm 2.9^{*}$ \\
\hline
\end{tabular}

AmpB-mediated cytotoxicity was determined using the MTS conversion assay (Promega Corp, Madison, WI), as previously described [23].

\subsection{Cytotoxicity assay on THP1 cells}

Human monocytic THP1 cells (ATCC TIB-202) were kindly provided by Dr JS Hill (St Paul's Hospital, UBC, Vancouver, BC). Cells were grown in complete RPMI without phenol red at $37^{\circ} \mathrm{C}\left(5 \% \mathrm{CO}_{2}\right)$ in $\mathrm{T} 75$ flasks. Before the cells reached a concentration of one million per $\mathrm{mL}$, the cells were transferred to either additional flasks or to 96-well plates and seeded at a density of 20,000 cells/well with the addition of PMA $(10 \mathrm{ng} / \mathrm{mL}$ final) to allow the cells to differentiate overnight. Twenty four hours post seeding, the differentiated cells were washed and the different concentrations of AmpB formulations were added (31 to $500 \mu \mathrm{g} / \mathrm{L}$ of AmpB) for $48 \mathrm{~h}$. After a $90 \mathrm{~min}$ incubation with the MTS reagent, percent AmpB-mediated cytotoxicity was determined 
using the MTS conversion assay (Promega Corp, Madison, WI), as previously described [23] or the LDH assay (Promega Corp, Madison, WI) as outlined by the manufacturer specifications. The MTS and LDH results were corrected by the amount of protein present in the respective wells. Purified LDH from the manufacturer kit was used as a positive control (data not shown).

\subsection{Minimum inhibitory concentration determination of the AmpB formulations on Candida growth in liquid medium}

RPMI without phenol red and supplemented with $10 \%$ Fetal bovine serum, 2\% dextrose plus penicillin and streptomycin was used to prepare dilutions of AmpB $(31.25,62.5,125,250$ and $500 \mu \mathrm{g} / \mathrm{L}$ - final drug concentration) from Fungizone ${ }^{\mathrm{TM}}$, Ambisome ${ }^{\mathrm{TM}}, \mathrm{iCo}-009$ and iCo-010 (AmpB concentration in the formulations was determined by HPLC). A vehicle control of iCo-009 and iCo-010 was used as a negative control. RPMI is the standard medium used in microplate drug susceptibility assays and is commonly used for testing of drug sensitivity for both Candida albicans and Cryptococcus neoformans [24]. A limitation of the RPMI medium is that there are frequent overlaps between the EC50 ranges for isolates that are putatively resistant or susceptible to Amphotericin B. The supplementation of RPMI medium with $2 \%$ glucose has lead to higher fungal growth rates and better separation of EC50s for isolates that are putatively resistant to fluconazole [21]. This is the methodology that we have used for our experiments.

The Candida albicans (ATCC reference strain 18804) inoculum was prepared as follows. The fungal cell concentration was determined using a hematocytometer. One hundred microlitres of a $5 \times 10^{5} / \mathrm{mL}$ preparation of Candida in RPMI was placed in the 96 well plates for a final concentration of $2.5 \times 10^{5}$ cells $/ \mathrm{mL}$. Each concentration was analyzed in eight replicates per experiment ( $\mathrm{n}=6-12$ independent experiments).

The plates were incubated for $48 \mathrm{~h}$ at $37^{\circ} \mathrm{C}$ protected from the light before analyzing the growth pattern using a spectrophotometer at $650 \mathrm{~nm}$. A new Candida agar plate was streaked each week to ensure the viability of the culture.

\footnotetext{
Acknowledgements

This work was supported by a grant-in-aid from iCo Therapeutics Inc., Vancouver, BC Canada and a Canadian Institutes of Health Research (CIHR) grant awarded to KMW.
}

\section{Author details}

'Division of Pharmaceutics and Biopharmaceutics, Faculty of Pharmaceutical Sciences, The University of British Columbia, Vancouver British Columbia, V6T 1Z3, Canada. ${ }^{2}$ Faculty of Environmental and Occupational Health, The University of British Columbia, Vancouver, British Columbia, V6T 1Z3, Canada. ${ }^{3}$ School of Health Sciences, British Columbia Institute of Technology, 3700 Willingdon Avenue, Burnaby, British Columbia, V5G 3H2, Canada. ${ }^{4} \mathrm{i}$ Co
Therapeutics Inc., 760-777 Hornby, Vancouver, British Columbia, V6Z 1S4, Canada.

\section{Authors' contributions}

CGL carried out the 293T cytotoxicity assays and drafted the manuscript. $J L$ carried out the THP1 cytotoxicity assays and the Candida efficacy experiments. KB participated in the design of the study. PG prepared the AmpB formulations and edited the manuscript. EKW designed and prepared the AmpB formulations. JZ prepared and characterized the novel AmpB formulations. JGC edited the manuscript. KMW participated in the design of the study and helped to draft the manuscript. All authors read and approved the final manuscript.

\section{Competing interests}

J. G. C. is an employee/co-founder/shareholder and director of iCo Therapeutics Inc. All other authors: none to declare.

Received: 15 July 2011 Accepted: 20 August 2011

Published: 20 August 2011

\section{References}

1. Calugi C, Trabocchi A, Guarna A: Novel small molecules for the treatment of infections caused by Candida albicans: a patent review 2. Expert Opin Ther Pat 2011, 21(3):381-97.

2. Marr KA: Fungal infections in oncology patients: update on epidemiology, prevention, and treatment. Curr Opin Oncol 2010, 22(2):138-42.

3. Rodriguez-Creixems M, Alcala L, Munoz P, Cercenado E, Vicente T, Bouza E: Bloodstream infections: evolution and trends in the microbiology workload, incidence, and etiology, 1985-2006. Medicine (Baltimore) 2008, 87(4):234-49.

4. Niimi M, Firth NA, Cannon RD: Antifungal drug resistance of oral fungi. Odontology 2010, 98(1):15-25.

5. Oura M, Sternberg TH, Wright ET: A new antifungal antibiotic, amphotericin B. Antibiot Annu 1955, 3:566-73.

6. Baginski M, Sternal K, Czub J, Borowski E: Molecular modelling of membrane activity of amphotericin $\mathrm{B}$, a polyene macrolide antifungal antibiotic. Acta Biochim Pol 2005, 52(3):655-8.

7. Sangalli-Leite F, Scorzoni L, Mesa-Arango AC, Casas C, Herrero E, Soares Mendes Gianinni MJ, Rodriguez-Tudela JL, Cuenca-Estrella M, Zaragoza O: Amphotericin B mediates killing in Cryptococcus neoformans through the induction of a strong oxidative burst. Microbes Infect 2011, 13(5):457-67.

8. Krcmery V, Barnes AJ: Non-albicans Candida spp. causing fungaemia: pathogenicity and antifungal resistance. J Hosp Infect 2002, 50(4):243-60.

9. Kelly SL, Lamb DC, Kelly DE, Manning NJ, Loeffler J, Hebart H, Schumacher $\mathrm{U}$, Einsele $\mathrm{H}$ : Resistance to fluconazole and cross-resistance to amphotericin B in Candida albicans from AIDS patients caused by defective sterol delta5,6-desaturation. FEBS Lett 1997, 400(1):80-2.

10. Barker KS, Crisp S, Wiederhold N, Lewis RE, Bareither B, Eckstein J, Barbuch R, Bard M, Rogers PD: Genome-wide expression profiling reveals genes associated with amphotericin $B$ and fluconazole resistance in experimentally induced antifungal resistant isolates of Candida albicans. $J$ Antimicrob Chemother 2004, 54(2):376-85.

11. Chamilos G, Kontoyiannis DP: Update on antifungal drug resistance mechanisms of Aspergillus fumigatus. Drug Resist Updat 2005, 8(6):344-58.

12. Safdar A, Ma J, Saliba F, Dupont B, Wingard JR, Hachem RY, Mattiuzzi GN, Chandrasekar PH, Kontoyiannis DP, Rolston KV, Walsh TJ, Champlin RE, Raad II: Drug-induced nephrotoxicity caused by amphotericin B lipid complex and liposomal amphotericin B: a review and meta-analysis. Medicine (Baltimore) 2010, 89(4):236-44.

13. Thornton SJ, Wasan KM: The reformulation of amphotericin B for oral administration to treat systemic fungal infections and visceral leishmaniasis. Expert Opin Drug Deliv 2009, 6(3):271-84.

14. Wasan KM, Wasan EK, Gershkovich P, Zhu X, Tidwell RR, Werbovetz KA, Clement JG, Thornton SJ: Highly effective oral amphotericin B formulation against murine visceral leishmaniasis. J Infect Dis 2009, 200(3):357-60.

15. Wasan EK, Gershkovich P, Zhao J, Zhu X, Werbovetz K, Tidwell RR, Clement JG, Thornton SJ, Wasan KM: A novel tropically stable oral 
amphotericin B formulation (iCo-010) exhibits efficacy against visceral Leishmaniasis in a murine model. PLoS Negl Trop Dis 2010, 4(12):e913.

16. Wasan EK, Bartlett K, Gershkovich P, Sivak O, Banno B, Wong Z, Gagnon J, Gates B, Leon CG, Wasan KM: Development and characterization of oral lipid-based amphotericin B formulations with enhanced drug solubility, stability and antifungal activity in rats infected with Aspergillus fumigatus or Candida albicans. Int J Pharm 2009, 372(1-2):76-84.

17. Razonable RR, Henault M, Lee LN, Laethem C, Johnston PA, Watson HL, Paya CV: Secretion of proinflammatory cytokines and chemokines during amphotericin B exposure is mediated by coactivation of toll-like receptors 1 and 2. Antimicrob Agents Chemother 2005, 49(4):1617-21.

18. Atkinson AJ Jr, Bennett JE: Amphotericin B pharmacokinetics in humans. Antimicrob Agents Chemother 1978, 13(2):271-6.

19. Sesana AM, Monti-Rocha R, Vinhas SA, Morais CG, Dietze R, Lemos EM: In vitro activity of amphotericin $B$ cochleates against Leishmania chagasi. Mem Inst Oswaldo Cruz 2011, 106(2):251-3.

20. Rex JH, Pfaller MA, Galgiani JN, Bartlett MS, Espinel-Ingroff A, Ghannoum MA, Lancaster M, Odds FC, Rinaldi MG, Walsh TJ, Barry AL: Development of interpretive breakpoints for antifungal susceptibility testing: conceptual framework and analysis of in vitro-in vivo correlation data for fluconazole, itraconazole, and candida infections. Subcommittee on Antifungal Susceptibility Testing of the National Committee for Clinical Laboratory Standards. Clin Infect Dis 1997, 24(2):235-47.

21. Rodriguez-Tudela JL, Martinez-Suarez JV: Improved medium for fluconazole susceptibility testing of Candida albicans. Antimicrob Agents Chemother 1994, 38(1):45-8.

22. Chang HC, Chang JJ, Chan SH, Huang AH, Wu TL, Lin MC, Chang TC: Evaluation of Etest for direct antifungal susceptibility testing of yeasts in positive blood cultures. J Clin Microbiol 2001, 39(4):1328-33.

23. Zager RA: Polyene antibiotics: relative degrees of in vitro cytotoxicity and potential effects on tubule phospholipid and ceramide content. Am $J$ Kidney Dis 2000, 36(2):238-49.

24. Anaissie EJ, Paetznick VL, Ensign LG, Espinel-Ingroff A, Galgiani JN, Hitchcock CA, LaRocco M, Patterson T, Pfaller MA, Rex JH, Rinaldi MG: Microdilution antifungal susceptibility testing of Candida albicans and Cryptococcus neoformans with and without agitation: an eight-center collaborative study. Antimicrob Agents Chemother 1996, 40(10):2387-91.

doi:10.1186/1476-511X-10-144

Cite this article as: Leon et al:: In vitro cytotoxicity of two novel oral formulations of Amphotericin B (iCo-009 and iCo-010) against Candida albicans, human monocytic and kidney cell lines. Lipids in Health and Disease 2011 10:144.

\section{Submit your next manuscript to BioMed Central and take full advantage of:}

- Convenient online submission

- Thorough peer review

- No space constraints or color figure charges

- Immediate publication on acceptance

- Inclusion in PubMed, CAS, Scopus and Google Scholar

- Research which is freely available for redistribution

Submit your manuscript at www.biomedcentral.com/submit
Biomed Central 